\title{
Orienteering performance and ingestion of glucose and glucose polymers
}

\author{
U.M. Kujala, $\mathrm{MD}^{1,2}$ O.J. Heinonen, $\mathrm{MD}^{1}$ M. Kvist, $\mathrm{MD}^{1}$, O-P. Kärkkäinen, $\mathrm{MSC}^{3} \mathrm{~J}$. \\ Marniemi, $\mathrm{PhD}^{2}$ K.Niittymäki, MSC ${ }^{2}$ and E. Havas, MSC $^{3}$
}

\author{
${ }^{1}$ Paavo Nurmi Centre, Sports Medical Research Unit, Turku, Finland \\ ${ }^{2}$ The Rehabilitation Research Centre of the Social Insurance Institution, Turku \\ ${ }^{3}$ Finnish Orienteering Association
}

\begin{abstract}
Summary
The benefit of glucose polymer ingestion in addition to 2.5 per cent glucose before and during a prolonged orienteering competition was studied. The final time in the competition in the group ingesting $\mathbf{2 . 5}$ per cent glucose (group G, $n=10$ ) was $113 \mathrm{~min} 37 \mathrm{~s} \pm 8 \mathrm{~min} 11 \mathrm{~s}$, and in the group which had additionally ingested glucose polymer (group G+GP, $\mathrm{n}=8) 107 \mathrm{~min} 18 \mathrm{~s} \pm 4 \mathrm{~min} 41 \mathrm{~s}$ (NS). One fifth (21 per cent) of the time difference between the two groups was due to difference in orienteering errors. Group G+GP orienteered the last third of the competition faster than group $G$ $(p<0.05)$. The time ratio between the last third of the competition and the first third of the competition was lower in group $G+G P$ than in group $G(p<0.05)$. After the competition, there was statistially insignificant tendency to higher serum glucose and lower serum free fatty acid concentrations in group $G+G P$, and serum insulin concentration was higher in group $G+G P$ than in group $G$ $(p<0.05)$. Three subjects reported that they exhausted during the competition. These same three subjects had the lowest serum glucose concentrations after the competition (2.9 mmol.1 $1^{-1}, 2.9 \mathrm{mmol}^{-1}, 3.5 \mathrm{mmol}^{-1}$ ) and all of them were from group $G$. It is concluded that glucose polymer syrup ingestion is beneficial for prolonged psychophysical performance.
\end{abstract}

Keywords: Glucose, glucose polymer, endurance, exercise, skill sports

\section{Introduction}

Orienteering is a sport in which the orienteer has to find his way through unfamiliar terrain from one control (checkpoint) to another with a map and a compass. The result of a competition thus, in addition to physical capacity, also depends on the level of planning and other orienteering skills as well as on the ability to maintain the concentration level. In top level orienteers the $\mathrm{O}_{2}$ consumption and heart rate are quite

Correspondence to: DR Urho Kujala, Paavo Nurmi Centre, Sport Medical Research Unit, Kiinamyllynkatu 10, SF-20520 Turku, Finland

(C) 1989 Butterworth \& Co (Publishers) Ltd 0306-3674/89/020105-04\$03.00 even and about 90 per cent of the maximal capacity during competitions ${ }^{1,2}$. Throughout a long competition, both the heart rate and the blood lactate concentration are higher than the anaerobic threshold determined in laboratory conditions ${ }^{2}$. The high heart rate during an orienteering competition may be explained by central stimuli ${ }^{3}$ in addition to the loading of several different muscle groups at the same time with uneven rhythm when running in difficult terrain.

Dietary carbohydrates and fluids during prolonged endurance exercise enhance performance. The two most important factors limiting prolonged, strenuous exercise are believed to be dehydration and depletion of muscle glycogen stores. During prolonged exercise a muscle can increase its net blood borne glucose uptake 10 - to 20 -fold above the resting value 4 . Decrease in blood glucose concentration and simultaneous decrease in prolonged physical performance level are observed, although the primary factors limiting performance are not known exactly ${ }^{5}$. The effect of lowered blood glucose levels on performance level in skill sports through inadequate nutrition of the brain is also discussed ${ }^{6}$. Glucose is the major energy source for the brain ${ }^{7}$. The brain cannot store this carbohydrate, which means that the cerebral metabolism is critically dependent upon freely circulating ambient blood glucose in order to maintain normal neuronal function ${ }^{8}$.

Gastric emptying is controlled for example by osmoreceptors in the duodenum ${ }^{9}$. Gastric emptying rate seems to be the primary limiting factor in delivering water, carbohydrate, and minerals to the body ${ }^{10}$, hyperosmolalic solutions being unfavourable. Glucose polymers appear to be favourable because their osmolality is low, while high amounts of carbohydrates are delivered into the intestine.

However, on the basis of recent studies, there is some disagreement whether carbohydrate feeding during prolonged exercise enhances performance by preventing the depletion of muscle glycogen stores $^{11,12}$. The greatest volume of the repeated bolus and its greatest carbohydrate concentration than can simultaneously deliver fluid and enhance performance is not known ${ }^{13}$. There is also wide individual variation in gastric emptying and intestinal transport 
and also in other responses to fluid and substrate administration.

In 1986 there were 3151 ranked orienteers in the male open class in Finland. We have analyzed the fluid and carbohydrate use of orienteers in the Finnish Championships and found it to be below the theoretically optimal level. The aim of this study was to analyze the benefit of more abundant energy and fluid ingestion than is commonly used before and during orienteering competition (prolonged psychophysical performance) by using glucose polymers.

\section{Subjects and methods}

Eighteen male top level orienteers volunteered as subjects for this study and gave their informed consent (Table 1). The subjects were ranked between 4 and 157 in the Finnish ranking list, based in success in orienteering competitions during previous months. The orienteers were randomly divided into two groups. Ten orienteers (group $G$ ) ingested before and during their competition a total of $900 \mathrm{ml}$ of 2.5 per cent glucose according to a given schedule (Table 2). Eight orienteers (group G+GP) ingested $900 \mathrm{ml}$ of 2.5 per cent glucose solution and additionally $400 \mathrm{ml}$ of glucose polymer syrup (Table 2). There was no significant difference in the mean of the ranking positions between the groups. Hence it was possible to obtain two groups of the same performance level for this study. All subjects orienteered the same course, which was 16000 metres long when measured from one control to another. The course had 24 control points and consisted of three different parts (Figure 1).

From start to control $8(4200 \mathrm{~m})$, difficult (detailed) orienteering

From control 8 to control 15 (7000 m), easy (rough) orienteering

From control 15 to the finish $(4800 \mathrm{~m})$, difficult (detailed) orienteering

Table 1. Age, height, weight and training of the athletes

\begin{tabular}{lrc}
\hline & $G(n=10)$ & $G+G P(n=8)$ \\
\hline Age (years) & $25.1 \pm 2.6$ & $26.3 \pm 3.2$ \\
Height $(\mathrm{cm})$ & $181.8 \pm 6.5$ & $182.7 \pm 4.9$ \\
Weight $(\mathrm{kg})$ & $69.7 \pm 5.7$ & $67.9 \pm 5.5$ \\
Training 1986 (hours/week) & $11.7 \pm 4.0$ & $10.8 \pm 2.6$ \\
\hline
\end{tabular}

G; group ingesting $2.5 \%$ glucose solution

$\mathrm{G}+\mathrm{GP}$; group ingesting $2.5 \%$ glucose solution and glucose polymer Mean \pm SD

Table 2. The study schedule

\begin{tabular}{lll}
\hline & \multicolumn{1}{c}{$G$} & $G+G P$ \\
\hline 60 min before start & blood sample A & blood sample A \\
55 min before start & $2.0 \mathrm{dl} 2.5 \%$ gluc. & $2.0 \mathrm{dl} 2.5 \%$ gluc. \\
& & $1.0 \mathrm{dl}$ gluc. polym. \\
30 min before start & $2.0 \mathrm{dl} 2.5 \%$ gluc. & $2.0 \mathrm{dl} 2.5 \%$ gluc. \\
& & $1.0 \mathrm{dl}$ gluc. polym. \\
15 min before start & $1.0 \mathrm{dl} 2.5 \%$ gluc. & $1.0 \mathrm{dl} 2.5 \%$ gluc. \\
Control $8 / 4.2 \mathrm{~km}$ & $2.0 \mathrm{dl} 2.5 \%$ gluc. & $2.0 \mathrm{dl} 2.5 \%$ gluc. \\
Control $15 / 11.2 \mathrm{~km}$ & $2.0 \mathrm{dl} 2.5 \%$ gluc. & $1.0 \mathrm{dl}$ gluc. polym. \\
& $2.0 \mathrm{dl} 2.5 \%$ gluc. \\
Finish $/ 16 \mathrm{~km}$ & blood sample B & $1.0 \mathrm{dl}$ gluc. polym. \\
\hline
\end{tabular}

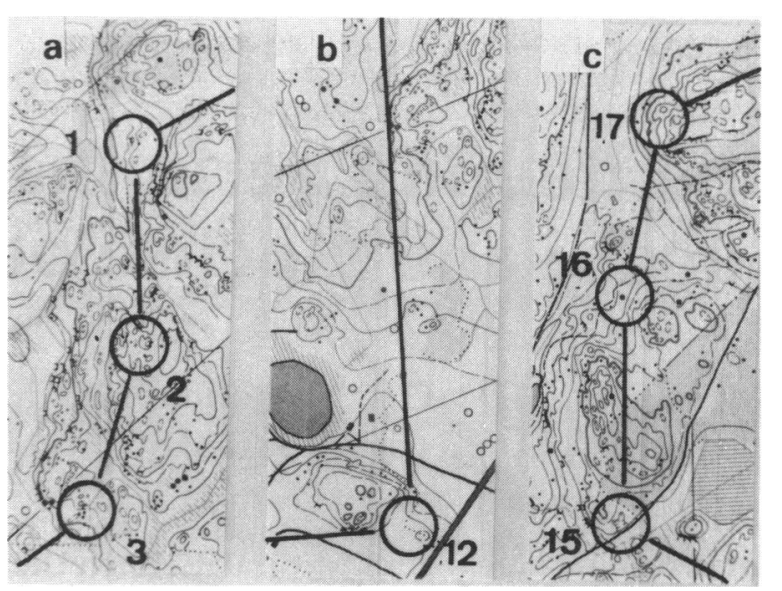

Figure 1. Examples from the different thirds of the orienteering course $a$ : Detailed orienteering from the first third of the race; $b$ : Rough orienteering from the second third of the race; $c$ : Detailed orienteering from the last third of the race

The air temperature varied during the competition between $+5.5^{\circ} \mathrm{C}$ and $+6.9^{\circ} \mathrm{C}$ and the relative humidity between 50 per cent and 60 per cent.

The glucose polymer (maltodextrin) consisted of 5-7 glucose units/molecule $\left(\mathrm{MX}^{\mathrm{R}}\right.$; Huhtamäki OY Marli, Finland). The glucose polymer syrup had $67 \mathrm{~g}$ carbohydrates in $100 \mathrm{ml}$ water and its osmolality was $674 \mathrm{mOsm} / \mathrm{kg}$ water. The average temperature of the liquids ingested was $20^{\circ} \mathrm{C}$. The scheme for group $\mathrm{G}$ was chosen to correspond to the normal practice among Finnish top level orienteers recorded in Finnish Championships in 1986. When adjusting the scheme of group G+GP, the practical possibilities in normal orienteering competition had to be taken into account.

Antecubital venous blood samples were taken one hour before the competition (sample A) and 60-120 s after the competition (sample B) (Table 2). Serum was separated and stored in $-20^{\circ}$ until analysis. Immunoreactive serum insulin (S-Ins) was determined by the commercial RIA-method (Novo, Denmark), and serum free fatty acids (S-FFA) were determined by enzymatic colorimetric method (Nefa C, Wako Chemicals $\mathrm{GmbH}$, West Germany). A Transcon 102 FN analyzer (Orion Analytica, Finland) was utilized for combined enzymatic determination of serum glucose (S-Gluc) $)^{14}$.

After the competition, the subjects answered a structured questionnaire including data on previous training and other background information, and comments on aspects such as orienteering errors made and gastrointestinal symptoms experienced during the study competition. They also marked the exact route that they had used on the maps. On the basis of this information the time lost due to orienteering errors was calculated. To evaluate the number of errors, the sum score of orienteering errors was formed as follows:

1 point; small error; time loss $30 \mathrm{~s}-2 \min 30 \mathrm{~s}$ 2 points; moderate error; $2 \mathrm{~min} 30 \mathrm{~s}-5 \mathrm{~min}$

3 points; major error; more than $5 \mathrm{~min}$

During the days preceding the study competition, the subjects had a normal Finnish mixed diet; none of the subjects underwent special carbohydrate loading. 
In the morning before the competition, the subjects had a normal mixed light breakfast according to their own habits, but including no more than $500 \mathrm{ml}$ of fluids.

Statistical differences were tested using the MannWhitney $U$ test and $X^{2}$ test.

\section{Results}

One subject from both groups suffered from gastrointestinal symptoms which lowered his performance. On the basis of subjective opinions, three subjects in group G and none in group G+GP reported feeling exhausted during the competition.

There was no time difference between the groups in the first third of the competition, but group G+GP ran the last third in a shorter time than the control group $G$ $(p<0.05)$, (Table 3). About one fifth (21 per cent) of the total time difference between the groups was due to difference in orienteering errors observed (Table 3). The sum score of the errors during the last third of the competition was $1.8 \pm 2.1$ in group $G$ and $1.4 \pm 1.3$ in group G+GP (NS). The time ratio between the last third of the competition and the first third of the competition was lower in group G+GP than in group $G$ $(\mathrm{p}<0.05)$, (Table 3).

After the competition, the serum insulin concentration was higher in group G+GP than in group G $(\mathrm{p}<0.05)$, (Figure 2). An insignificant trend to higher serum glucose and lower serum free fatty acid concentrations were correspondingly observed (Figures 3 and 4).

The lowest glucose concentration after the competition in group G+GP was 4.8 mmol. $\mathrm{l}^{-1}$, while in group $G$ there were two subjects with a glucose concentration of $2.9 \mathrm{mmol} . \mathrm{l}^{-1}$ and one subject of $3.5 \mathrm{mmol} . \mathrm{l}^{-1}$. These three subjects orienteered the last third of the competition proportionally more slowly than the mean of group $\mathrm{G}$, and they were also the same subjects who reported that they had become exhausted during the competition.

\section{Discussion}

Our study scheme was not assessed to be theoretically optimal with reference to fluid and energy ingestion, but the practical possibilities in an orienteering competition has to be taken into account. For example, the ingestion of carbohydrates 45-15 minutes before exercise, which is common in orienteers, is not recommended because it can cause hyperinsulinemia followed

Table 3. The time during the different thirds of the competition, the time ratio between the last and first third of the competition, the elapsed time and the estimated time lost due to orienteering errors during different thirds of the competition in groups $G$ and $\mathrm{G}+\mathrm{GP}$ (mean $\pm \mathrm{SD})\left({ }^{*} \mathrm{P}<0.05\right.$, difference between the groups)

\begin{tabular}{lcc}
\hline & $G(n=10)$ & $G+G P(n=8)$ \\
\hline Time I Start to control 8 (min.s) & $31.07 \pm 1.48$ & $30.52 \pm 1.39$ \\
Time II Control 8 to control 15 (min.s) & $42.05 \pm 2.10$ & $41.27 \pm 2.43$ \\
Time III Control 15 to finish (min.s) & $40.25 \pm 6.40$ & $34.59 \pm 1.59^{*}$ \\
Time III/time I & $1.30 \pm 0.21$ & $1.14 \pm 0.08^{*}$ \\
Total time (min.s) & $113.37 \pm 8.11$ & $107.18 \pm 4.41$ \\
Time lost due to errors 1 (min.s) & $2.27 \pm 2.00$ & $2.11 \pm 1.33$ \\
Time lost due to errors 2 (min.s) & $0.36 \pm 0.56$ & $0.23 \pm 0.45$ \\
Time lost due to errors 3 (min.s) & $1.56 \pm 2.50$ & $1.05 \pm 1.15$ \\
\hline
\end{tabular}

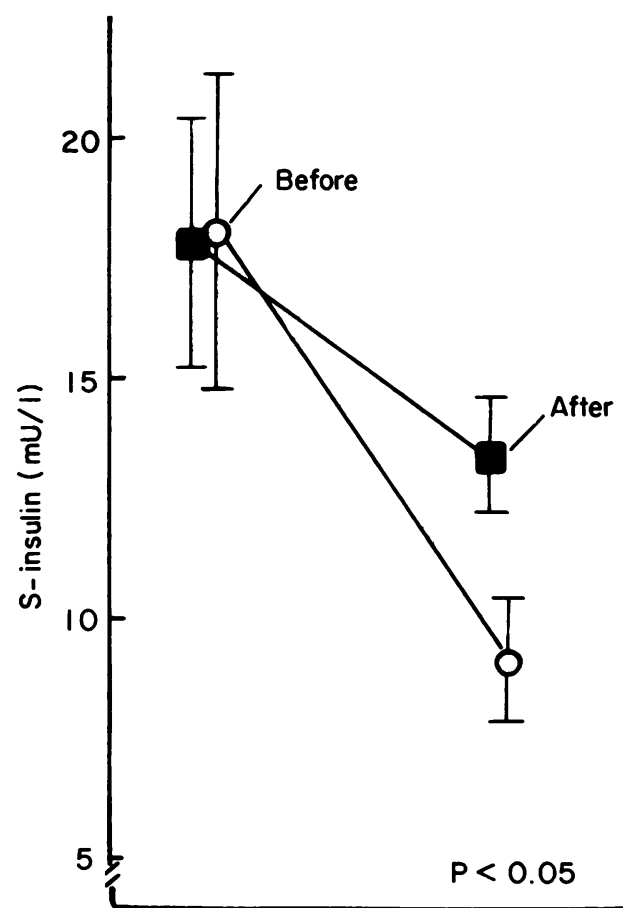

Figure 2. Serum insulin (S-Ins) concentration (mean \pm SEM) 60 min before and immediately after the competition

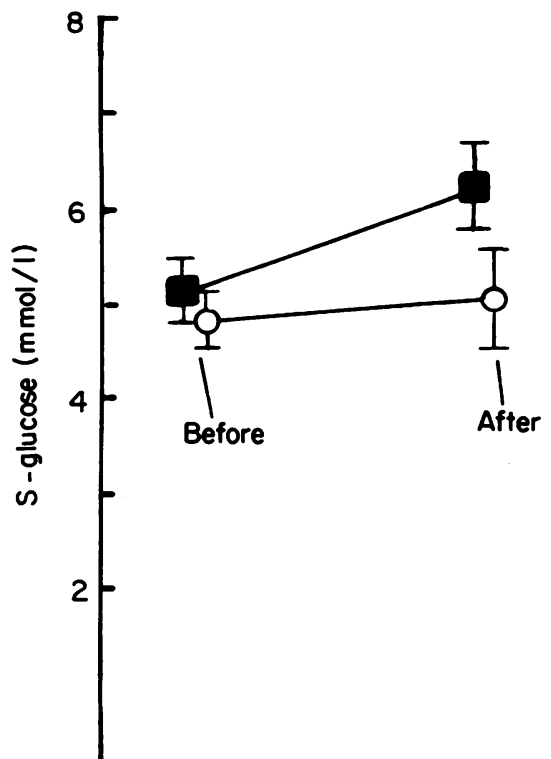

Figure 3. Serum glucose (S-Gluc) concentration (mean \pm SEM) $60 \mathrm{~min}$ before and immediately after the competition

by hypoglycemia ${ }^{15}$. In our study, pre-exercise carbohydrate ingestion did not cause significant decrease of endurance capacity in group G+GP when compared to group F (Table 3). The group G+GP was drinking $200 \mathrm{ml}$ of a 2.5 per cent glucose solution and $100 \mathrm{ml}$ of a 67 per cent carbohydrate solution at each time point except for one. This effectively gives $300 \mathrm{ml}$ of a carbohydrate solution with a carbohydrate content of $24 \mathrm{gm} / 100 \mathrm{ml}$. On the basis of laboratory experiments, such a solution should be emptied reasonably rapidly from the stomach and should be reasonably well absorbed by the small intestine ${ }^{15}$.

There seems to be a more significant difference between the groups in the final results of the orienteering competition than in similar studies demanding only physical performance capacity ${ }^{12}$, although a dif- 


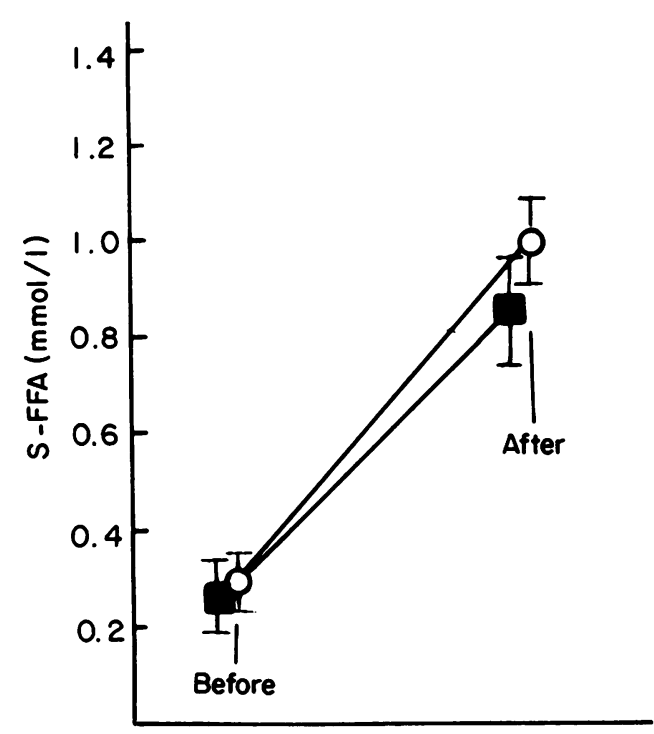

Figure 4. Serum free fatty acids (S-FFA) concentration (mean \pm SEM) 60 min before and immediately after the competition

ference in physical performance level has been noted during exercise exceeding 90 min exercise also earlier ${ }^{5}$. There is always a problem in attempting to compare the performance of two different subjects.

Because we can also compare the results of the last and the first thirds of the competition within both groups, we can exclude the possibility that the time differences between the groups are solely due to groups selection. The number of orienteering errors did not significantly differ between the groups, and the group difference in the time lost due to orienteering errors accounted for only one fifth of the total time difference between the two groups. We suggest that top level orienteers can concentrate and avoid making serious errors even when the brain is working on limited glucose. Thus one possible explanation for the significant difference between the results can be attributed to better nutrition of the brain. This is also in agreement with the fact that those subjects whose blood glucose concentrations were lowest at the finish subjectively became exhausted and objectively had longer elapsed times during the last third of the competition. This theory also agrees with other earlier studies in skill sports but more studies are required ${ }^{6}$.

It has been suggested that, when the initial glycogen levels are elevated, carbohydrate ingestion during exercise does not result in a significant saving of muscle glycogen or performance improvement during a two-hour exercise period ${ }^{11}$. We suggest that adequate carbohydrate ingestion using glucose polymers during long competitions is beneficial. In addition to increasing performance level, the use of glucose polymers might also hasten recovery, which is supported by a smaller decrease in S-Ins concentration, and also by a smaller increase in the plasma vasopressin concentration in group $G+G P$ than in group $G(p<0.001)$, which was recorded in the association with this study ${ }^{16}$. We did not measure muscle glycogen concentration, because taking the samples would have significantly disturbed the performance of the subjects in our study schedule, a fact we discovered in our pilot study.

We conclude that the ingestion of glucose polymer with 2.5 per cent glucose solution as one alternative in carbohydrate feeding during high level prolonged psychophysical performance is well tolerated and beneficial. On the basis of our study we recommend that more refreshment controls be provided in orienteering competitions.

\section{Acknowledgements}

The authors thank the Finnish Central Sports Federation and the Finnish Orienteering Association for their financial support. Our acknowledgement is also due to Erkki Alanen for the statistical work in the study.

\section{References}

1 Eklund, B., Hulten, B., Lundin, A., Nord, L., Saltin, B. and Silander, L. (Eds.) 'Orienteering' Trygg-Hansa, Stockholm, 1973

2 Kärkkäinen, O.P. (Ed.) 'Analysis of orienteering competition' (in Finnish) Faculty of Physical and Health Education, University of Jyväskylä, Jyväskylä, 1986

3 Morgan, W.P. Psychogenic factors and exercise metabolism: a review Med Sci Sports Exerc 1985, 17, 309_ 316

4 Wahren, J., Felig, P., Ahlborg, G. and Jorfeldt L. Glucose metabolism during leg exercise in man J Clin Invest 1971, 50, 2715-2725

5 Ivy, J.L., Miller, W. and Dover, V. Endurance improved by ingestion of a glucose-polymer supplement Med Sci Sports Exerc 1983, 15, 466-471

6 Shephard, R.J. and Leatt, P. Carbohydrate and fluid needs of the soccer player Sports Med 1987, 4, 164-176

7 Arky, R.A. 'Hypoglycemia' In Endocrinology (vol. 2) G.F. Cahill, W.D. Odell, L. Martini, J.T. Potts, D.H. Nelson, E. Steinberger, A.I. Winegrad (Eds.), Grune and Stratton, New York, 1979

8 Ingvar, D.J. and Lassen, N.A. (Eds.) 'Brain work: The coupling of function, metabolism and blood flow in the brain' Academic Press, New York, 1975

9 Hunt, J.N. and Knox, M.T. 'Regulation of gastric emptying' In Handbook of physiology (vol. 4) C.F. Code (Ed.), American Physiological Society, Washington DC, 1968

10 Wheeler, K.B. and Banwell, J.G. Intestinal water and electrolyte flux of glucose-polymer electrolyte solutions Med Sci Sports Exerc 1986, 18, 436-439

11 Flynn, M.G., Costill, D.L., Hawley, J.A., Fink, W.J., Neufer, P.D., Fielding, R.A. and Sleeper, M.D. Influence of selected carbohydrate drinks on cycling performance and glycogen use Med Sci Sports Exerc 1987, 19, $37-40$

12 Noakes, T.D., Lambert, E.V., Lambert, M.I., McArthur, P.S.,Myburgh, K.H. and Benade, A.J.S. Carbohydrate ingestion and muscle glycogen depletion during marathon and ultramarathon racing Eur J App Physiol 1988, 57, 482-489

13 Mitchell, J.B., Costill, D.L., Houmard, J.A., Flynn, M.G., Fink, W.J. and Beltz J.D. Effects of carbohydrate ingestion on gastric emptying and exercise performance Med Sci Sports Exerc 1988, 20, 110-115

14 Lowry, O.H. and Passonneau, J.V.(Eds.) 'Flexible system of enzymatic analysis' Academic Press, New York, 1972

15 Costill, D.L. Carbohydrates for exercise; dietary demands for optimal performance Int J Sports Med 1988, 9, $1-18$

16 Viinamäki, O., Heinonen, O.J., Kujala, U.M. and Alen, M. 1988 Glucose polymer syrup attenuates strenuous physical exercise-induced vasopressin release Acta Physiol Scand 1988, 134,(575), 103 